Journal for ImmunoTherapy of Cancer

\section{HITM-SURE: Hepatic immunotherapy for metastases phase Ib anti-CEA CAR-T study utilizing pressure enabled drug delivery}

To cite: Katz SC, Moody AE, Guha P, et al. HITM-SURE: Hepatic immunotherapy for metastases phase Ib anti-CEA CAR-T study utilizing pressure enabled drug delivery. Journal for ImmunoTherapy of Cancer 2020;8:e001097. doi:10.1136/ jitc-2020-001097

Accepted 22 July 2020

Check for updates

(C) Author(s) (or their employer(s)) 2020. Re-use permitted under CC BY-NC. No commercial re-use. See rights and permissions. Published by BMJ.

${ }^{1}$ Surgery, Roger Williams Medical Center, Providence, Rhode Island, USA

${ }^{2}$ Medicine, Roger Williams Medical Center, Providence, Rhode Island, USA

${ }^{3}$ Surgery, Boston University School of Medicine, Boston, Massachusetts, USA

${ }^{4}$ Radiology, Roger Williams Medical Center, Providence, Rhode Island, USA

${ }^{5}$ Pathology, Roger Williams Medical Center, Providence,

Rhode Island, USA

${ }^{6}$ Research, University of Colorado Denver School of Medicine, Aurora, Colorado, USA

${ }^{7}$ Sorrento Therapeutics Inc, San

Diego, California, USA

${ }^{8}$ IT Bio, LLC, Boston,

Massachusetts, USA

Correspondence to

Dr Steven C Katz;

skatz@chartercare.org

\section{ABSTRACT}

In recent years, cell therapy technologies have resulted in impressive results in hematologic malignancies. Treatment of solid tumors with chimeric antigen receptor T-cells (CAR-T) has been less successful. Solid tumors present challenges not encountered with hematologic cancers, including high intra-tumoral pressure and ineffective CAR-T trafficking to the site of disease. Novel delivery methods may enable CAR-T therapies for solid tumor malignancies. A patient with liver metastases secondary to pancreatic adenocarcinoma received CAR-T targeting carcinoembryonic antigen (CEA). Previously we reported that Pressure-Enabled Drug Delivery (PEDD) enhanced CAR-T delivery to liver metastases 5.2-fold. Three doses of anti-CEA CAR-T were regionally delivered via hepatic artery infusion (HAl) using PEDD technology to optimize the therapeutic index. Interleukin-2 was systemically delivered by continuous intravenous infusion to support CAR-T in vivo. HAI of anti-CEA CAR-T was not associated with any serious adverse events (SAEs) above grade 3 and there were no on-target/ off-tumor SAEs. Following CAR-T treatment, positron emission tomography-CT demonstrated a complete metabolic response within the liver, which was durable and sustained for 13 months. The response was accompanied by normalization of serum tumor markers and an abundance of CAR+ cells found within post-treatment tumor specimens. The findings from this report exhibit biologic activity and safety of regionally infused CAR-T for an indication with limited immune-oncology success to date. Further studies will determine how HAl of CAR-T may be included in multidisciplinary treatment plans for patients with liver metastases. ClinicalTrials.gov number, NCT02850536.

\section{BACKGROUND}

Liver metastases (LM) are a common cause of mortality in patients with solid tumor malignances. ${ }^{1}$ Increased intra-tumoral pressure, inefficient chimeric antigen receptor
T-cells (CAR-T) trafficking, and the immunosuppressive microenvironment inherent to the liver are three of the major barriers to effective therapy. ${ }^{2-4}$ We have developed regional cell therapy delivery approaches to address the unique challenges associated with the treatment of solid tumors with CAR-T. We present evidence of potential therapeutic benefit of adoptive T-cell therapy against LM with the use of CAR-T targeting carcinoembryonic antigen (CEA) in a single subject. Two previous phase $1 / 1 \mathrm{~b}$ clinical trials evaluated regional administration of anti-CEA CAR-T directly into the liver employing hepatic artery infusion (HAI) for the treatment of CEAexpressing LM. ${ }^{14}$ These trials confirmed the safety of regional liver CAR-T infusion and provided promising signals of clinical activity using CEA-directed cell therapy products, warranting further investigation. In this study, we tested the safety and feasibility of combining regional CAR-T infusions for LM with a device designed to overcome high intra-tumoral pressures.

\section{CASE PRESENTATION}

A man in his early 50 s presented with poorly differentiated pancreatic adenocarcinoma and synchronous LM. Immunohistochemical (IHC) stains confirmed expression of CK7 and CA 19-9, while mismatch repair protein expression was intact. CK20, CDX-2, TTF-1, and synaptophysin were also negative by IHC stains. The patient's Eastern Cooperative Oncology Group (ECOG) performance status was 0 and he received first-line standard-of-care systemic chemotherapy with 5-Fluorouracil (5-FU), 
leucovorin, irinotecan, and oxaliplatin (FOLFIRINOX). He tolerated FOLFIRINOX well with only grade 1 and grade 2 side effects, including nausea, diarrhea, alopecia, fatigue, and hyperglycemia. After nine cycles of FOLFIRINOX, a restaging CT scan demonstrated persistent disease in the liver and the pancreas. The patient did not receive any other therapies prior to systemic chemotherapy. Following confirmation of eligibility, the patient was enrolled in this clinical trial of anti-CEA-targeted CAR-T for persistent metastatic disease in the liver.

\section{METHODS}

\section{Study design}

This open label, phase 1, fixed-dose study was designed to evaluate the use of second-generation anti-CEA CAR-T delivered directly into the liver by HAI using Pressure-Enabled Drug Delivery (PEDD) technology for patients with treatment-refractory CEA-expressing LM. Patients exhibiting low-volume extrahepatic disease in the abdomen and/or lungs were eligible. The enrollment target was five subjects. Each treatment period was conducted over 10 weeks which allowed for leukapheresis, CAR-T production, 3 CAR-T HAI at weekly intervals $\left(1 \times 10^{10}\right.$ cells/ patient), and on-trial follow-up. Low-dose continuous intravenous infusion (CIVI) with interleukin-2 (IL-2) $(50,000 \mathrm{IU} / \mathrm{kg} /$ day $)$ was administered for 4 weeks during the CAR-T infusion period. Clinical response and survival were measured from the time of patient enrollment.

\section{CAR-T production}

Autologous peripheral blood mononuclear cells (PBMCs) were isolated by leukapheresis and activated with anti-CD3 (OKT3 clone) antibody for 48 hours. Post-activation, cells were retrovirally transduced with a construct encoding an anti-CEA scfv-CD28/CD3 $\zeta$ CAR. Three rounds of spinoculation were performed to transduce T-cells in the presence of retronectin. ${ }^{4}$ CAR-T were expanded to exceed a threshold of $3 \times 10^{10}$ cells, with storage of additional doses when feasible. Transduction efficiency was determined 72 hours post transduction and in vitro cytotoxicity assays were performed as previously described, ${ }^{5}$ which confirmed that the CAR-T product specifically lysed CEA+ tumor cells (specific lysis 29\% at effector:target ratio of 10:1).

\section{Correlative studies}

All blood draws occurring on days of HAI were drawn prior to the procedure. Baseline tissue biopsies were collected immediately prior to HAI 1 while HAI 3 and HAI 4 biopsies were collected immediately post HAI. Quantification of plasma cytokines was performed using a MESO QuickPlex SQ 120 (Meso Scale Diagnostics LLC, Rockville, MD, USA). Immunofluorescence (IF) microscopy was performed on formalin-fixed paraffin-embedded tissue stained for 4',6-diamidino-2-phenylindole (DAPI), CEA, CD3, and CAR (CEA-Fc, TNK Therapeutics, San Diego, CA, USA). Flow cytometry for circulating CAR-T was performed on PBMCs stained for CD3 and CAR. CAR DNA was quantified by qPCR using CAR-specific primers and normalized using primers specific for beta-2 microglobulin. Liver tumor tissue was homogenized in radioimmunoprecipitation assay (RIPA) buffer and analyzed by western blot for cytokine levels as previously described. ${ }^{5}$

\section{CAR-T HAI procedure}

CAR-T HAI was achieved via a trans-femoral, percutaneous approach using PEDD devices and was performed in the interventional radiology suite with moderate sedation. Prior to the first infusion, baseline (BL) biopsies were obtained. Three HAIs were carried out at weekly intervals using a super-selective delivery approach based on patient anatomy and tumor burden distribution. Low-dose, systemic IL-2 CIVI was initiated following the first CAR-T dose. During the third HAI session, repeat biopsies were performed. Lowdose IL-2 CIVI continued for 2 weeks following the completion of the CAR-T infusion period. During the reminder of on-trial follow-up, the patient returned to clinic at scheduled intervals. Six weeks following the final CAR-T HAI, post-treatment imaging was performed (figure 1A).

\section{RESULTS \\ Safety and adverse event profile}

HAIs of $1 \times 10^{10}$ anti-CEA CAR-T product cells were delivered to this subject with $63.4 \%$ expressing the CAR. The CAR-T treatments were not associated with any serious adverse events (SAEs) greater than grade 3 (table 1). Adverse events, grades 1-3 that were possibly attributed to therapy, having occurred within 1 month, included fevers, chills/rigors, tachycardia, hypotension, diarrhea, dehydration, fatigue, mild abdominal distension, edema, myalgias, decreased appetite, thrombocytopenia, electrolyte dysfunction, coagulopathies, and transient elevations in liver function tests (table 1). Symptoms were managed conservatively with ibuprofen, acetaminophen, intravenous fluid, and IL-2 dose reduction, as needed.

\section{Clinical response}

At the time of treatment, the patient presented with persistent, poorly differentiated pancreatic adenocarcinoma LM resistant to conventional chemotherapy. The primary tumor remained in place. Six weeks following CAR-T infusions, a positron emission tomography (PET) scan was performed which revealed no evidence of metabolically active disease in the liver or elsewhere in the body (figure 1B). Importantly, the patient's pretreatment PET scan exhibited several fluorodeoxyglucose (FDG) avid liver lesions. A CT scan with intravenous 
A

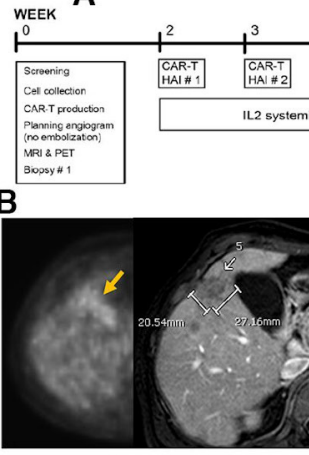

Baseline: 4/21/17

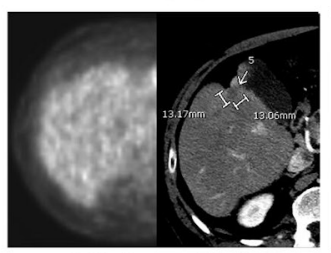

$2^{\text {nd }}$ Follow-up 8/29/17

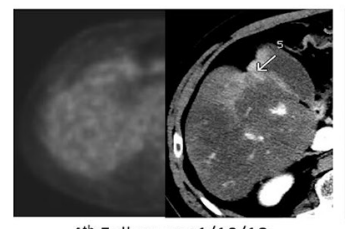

$4^{\text {th }}$ Follow-up: $1 / 10 / 18$

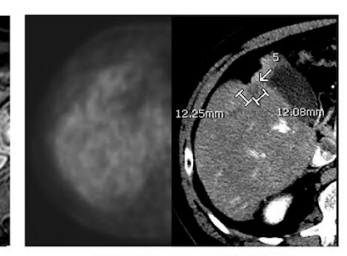

$1^{\text {st }}$ Follow-up: 6/20/17

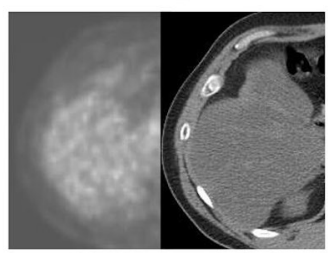

$3^{\text {RD }}$ Follow-up 11/21/17

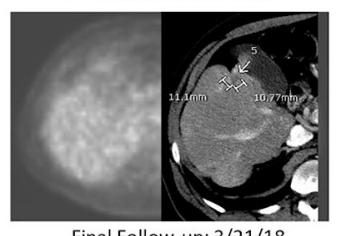

Final Follow-up: 3/21/18
C

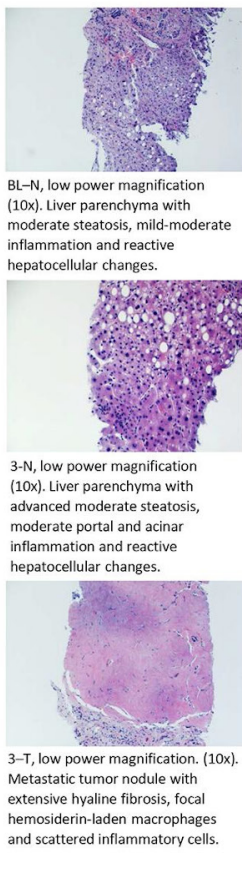

D

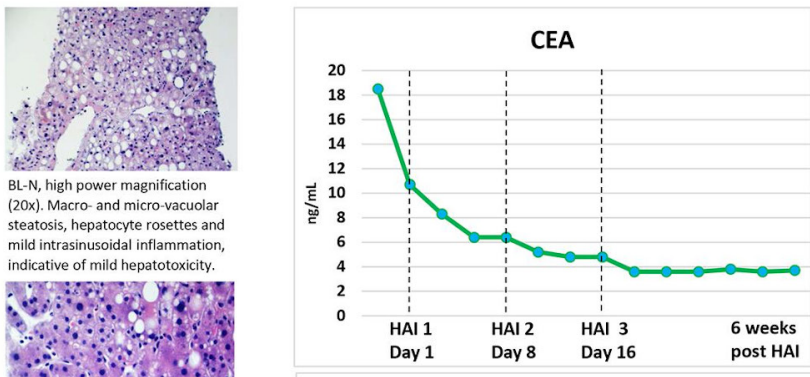

CA 19-9

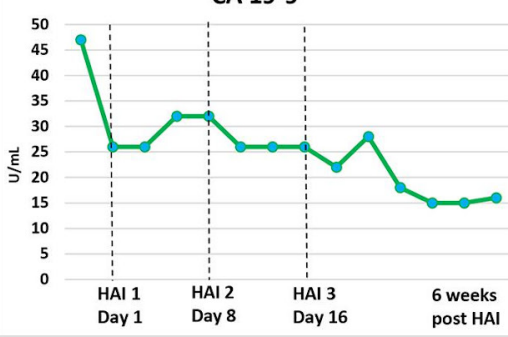

Figure 1 (A) Trial schematic. (B) Sequential imaging demonstrates complete metabolic response to intrahepatic CAR-T therapy. Each imaging time point is depicted with an image from a contrast-enhanced CT of the abdomen and a corresponding attenuation corrected PET image. The lesion, which is located adjacent to the gallbladder at the border between segments 4B and 5, is FDG avid at baseline and shows no activity on the first follow-up scan or any of the later time points. RECIST assessment of the lesion on CT shows partial response from baseline to the first time point, and stable disease from that time on. Note that there is increased peri-lesional enhancement on January 10,2018; this is likely due to a transient perfusion abnormality and the timing of image acquisition in relationship to contrast administration. (C) H\&E stain of non-neoplastic liver parenchyma $(\mathrm{N})$ at baseline and HAI 3, and metastatic tumor (T) collected at HAI 3. (D) Serum CEA and CA 19-9 were trended throughout the course of the study. Both tumor markers exhibited a continuous downward trend before normalizing following CAR-T HAI. This biochemical response correlated with the radiographic imaging, in addition to the post-treatment pathologic findings. CAR-T, chimeric antigen receptor T-cells; CEA, carcinoembryonic antigen; HAI, hepatic artery infusion; IL, interleukin; $\mathrm{PET}$, positron emission tomography; FDG, fluorodeoxyglucose.

contrast exhibited stable to slightly reduced size of target lesions, with no evidence of new metastatic lesions. Repeat imaging was performed 1 month later which revealed identical findings. A PET scan performed 3.7 months following treatment completion demonstrated a continued absence of metabolic activity within the liver, but there was an increase in metabolic activity at the primary site at the pancreatic head. The patient received radiation therapy to the pancreatic mass with 5-FU as a radiosensitizer. A follow-up PET scan exhibited a complete metabolic response in the primary tumor. The patient's liver response following CAR-T infusion was durable for 13 months before progression was evident at a single focus in the liver.

Pathologic analysis of the liver tumor biopsy specimens following CAR-T infusions revealed extensive hyaline fibrosis without evidence of injury to normal liver tissue (figure 1C). The patient's CEA and CA 19-9 tumor marker trends correlated with the radiographic imaging and pathologic findings. These markers were monitored throughout the trial and when using on-study tumor marker nadir as a reference point, the patient exhibited an $81 \%$ decrease in CEA and a 68\% decrease in CA $19-9$. Following anti-CEA CAR-T infusions, the patient's CEA and CA 19-9 tumor marker levels normalized for 2-3 months (figure 1D). It was during the time of the primary pancreatic tumor progression that these values increased beyond the normal range.

Given the profound intrahepatic response following the initial treatment with anti-CEA CAR-T, a fourth dose of $1 \times 10^{10}$ anti-CEA CAR-T, without IL-2 support, was delivered to address intrahepatic progression 13.8 months following enrollment. We confirmed ongoing CEA expression by IF on a biopsy specimen to exclude antigen loss as a mechanism for tumor resistance to the CAR-T (figure 2B). A post-infusion CT scan exhibited the recurrent segment 2 lesion, along with additional lesions in segments 4 and 7 . The corresponding PET scan revealed only a single focus of metabolic activity within segment 2 of the liver, with an SUV ${ }^{\mathrm{MAX}}$ of 8.0 (previously 6.3). There was minimal uptake within the primary tumor in the pancreas without evidence of new metastatic activity. 


\begin{tabular}{|c|c|}
\hline Grade* & Adverse event \\
\hline 1 & Abdominal distension \\
\hline 1 & $\uparrow$ Alkaline phosphatase \\
\hline 1 & $\uparrow A L T$ \\
\hline 1 & 个aPTT \\
\hline 1 & $\uparrow A S T$ \\
\hline 1 & Chills \\
\hline 3 & Dehydration \\
\hline 2 & Diarrhea \\
\hline 1 & Edema (LE) \\
\hline 2 & Fatigue \\
\hline 3 & Fevert \\
\hline 2 & Hyperbilirubinemia \\
\hline 3 & Hyperglycemia \\
\hline 3 & Hypertension \\
\hline 2 & Hypoalbuminemia \\
\hline 3 & Hypokalemia \\
\hline 1 & Hypomagnesemia \\
\hline 3 & Hyponatremia \\
\hline 3 & Hypophosphatemia \\
\hline 2 & Hypotension \\
\hline 1 & 个INR \\
\hline 2 & Pulmonary edema \\
\hline 2 & Rigors \\
\hline 1 & Tachycardia \\
\hline 1 & Thrombocytopenia \\
\hline
\end{tabular}

*Highest grade experienced for each event noted. †Led to $25 \%$ IL-2 dose reduction.

ALT, alanine aminotransferase; aPTT, activated partial thromboplastin time; AST, aspartate aminotransferase; IL-2, interleukin-2; INR, international normalized ratio.

Given the single site of uptake noted on PET, liverdirected therapy with microwave ablation was performed 3 weeks later. Six weeks following the ablation, re-imaging revealed new liver, lung, and peritoneal metastases for which the patient resumed systemic chemotherapy. The patient progressed through FOLFIRINOX followed by gemcitabine/abraxane, at which point he was referred for Yttrium-90 radioembolization therapy. Left liver radioembolization was performed 6 months following the initiation of systemic chemotherapy. The patient continued to decline before expiring secondary to disease progression, 23.2 months following trial enrollment. The total duration of the complete metabolic response of metastatic disease was 13 months.

\section{CAR-T trafficking and systemic inflammatory response}

LM biopsies were analyzed by IF and qPCR for CAR-T presence. CAR-T were detected by IF in LM samples post HAI 3 and 4 (figure 2A). CAR DNA was detected by qPCR in both normal liver and LM, with 215.3 CAR copies $/ 10^{4}$ cells post HAI 3 and 7.9 CAR copies $/ 10^{4}$ cells post HAI 4 in LM while normal liver tissue contained 535.6 CAR copies $/ 10^{4}$ cells post HAI 3 and 355.5 CAR copies $/ 10^{4}$ cells post HAI 4 .

PBMCs were collected at multiple trial time points for the assessment of circulating CAR-T which were quantified using qPCR and flow cytometry. Circulating CAR-T concentrations were below the level of detection by flow cytometry for all trial time points. Circulating CAR DNA reached a maximum level of $165.7 \mathrm{CAR}$ copies $/ 10^{4}$ PBMCs on treatment day (TD) 7 before it declined to less than 10 CAR copies $/ 10^{4}$ PBMCs on TD 15 , and remained at this low level through TD 43. There was no clinical evidence of on-target/off-tumor adverse events.

Protein lysates of liver tumor tissue collected at BL, infusion 3 , infusion 4 , and 48 days post infusion 4 were analyzed by western blot for indoleamine 2, 3-dioxygenase (IDO), PD-L1, IL-6, IL-17A, granulocyte-macrophage colony-stimulating factor (GM-CSF), and granulocytemacrophage colony-stimulating factor receptor (GM-CSF-R), using glyceraldehyde 3-phosphate dehydrogenase (GAPDH) as a loading control and densitometry plots (figure 2C). Relative to expression in pre-treatment biopsies, there was a decrease in protein expression of IDO, PD-L1, IL-17A, and GM-CSF in tumor tissue at the time of infusion 3. Compared with the progressive decline in protein expression of IDO, PD-L1, and GM-CSF in tumor tissue after CAR-T treatment, IL-17A protein expression was also lower, but remained fairly constant from infusion 3 through 48 days after infusion 4. IL-6 and GM-CSF-R expression increased from BL to infusion 3 but was similar to BL expression in specimens obtained 48 days after infusion 4 .

Plasma was analyzed for cytokines interferon gamma (IFNY), tumor necrosis factor alpha (TNF $\alpha$ ), IL-2, IL-6, IL-8, and IL-10 (figure 3). Plasma IFN $\gamma$ spiked on TD 8 to $1048 \mathrm{pg} / \mathrm{mL}$ but declined to BL levels by TD 16. Plasma levels of IL-6 and IL-8 both spiked on TD 45 to $24 \mathrm{pg} /$ $\mathrm{mL}$ and $351 \mathrm{pg} / \mathrm{mL}$, respectively. Plasma TNF $\alpha$ and IL-10 remained relatively low with $\mathrm{TNF} \alpha$ reaching a maximum concentration of $11 \mathrm{pg} / \mathrm{mL}$ on TD 8 and TD 45 and IL-10 reaching a maximum concentration of $13 \mathrm{pg} / \mathrm{mL}$ on TD 8. Plasma IL-2 remained around $10,500 \mathrm{pg} / \mathrm{mL}$ during the treatment period, then declined to BL levels on TD 30 , after systemic IL-2 infusions were discontinued, but spiked to $4384 \mathrm{pg} / \mathrm{mL}$ on TD 60,327 days prior to the fourth CAR-T HAI.

\section{DISCUSSION}

The liver is the most common site of hematogenous spread from gastrointestinal and hepatobiliary carcinomas, with many patients presenting with LM at the time of initial diagnosis. ${ }^{6}$ Although there have been many advancements in the management of LM in recent years, they are still a leading cause of death for the afflicted population. ${ }^{6}$ Inefficient CAR-T trafficking, high intra-tumoral 

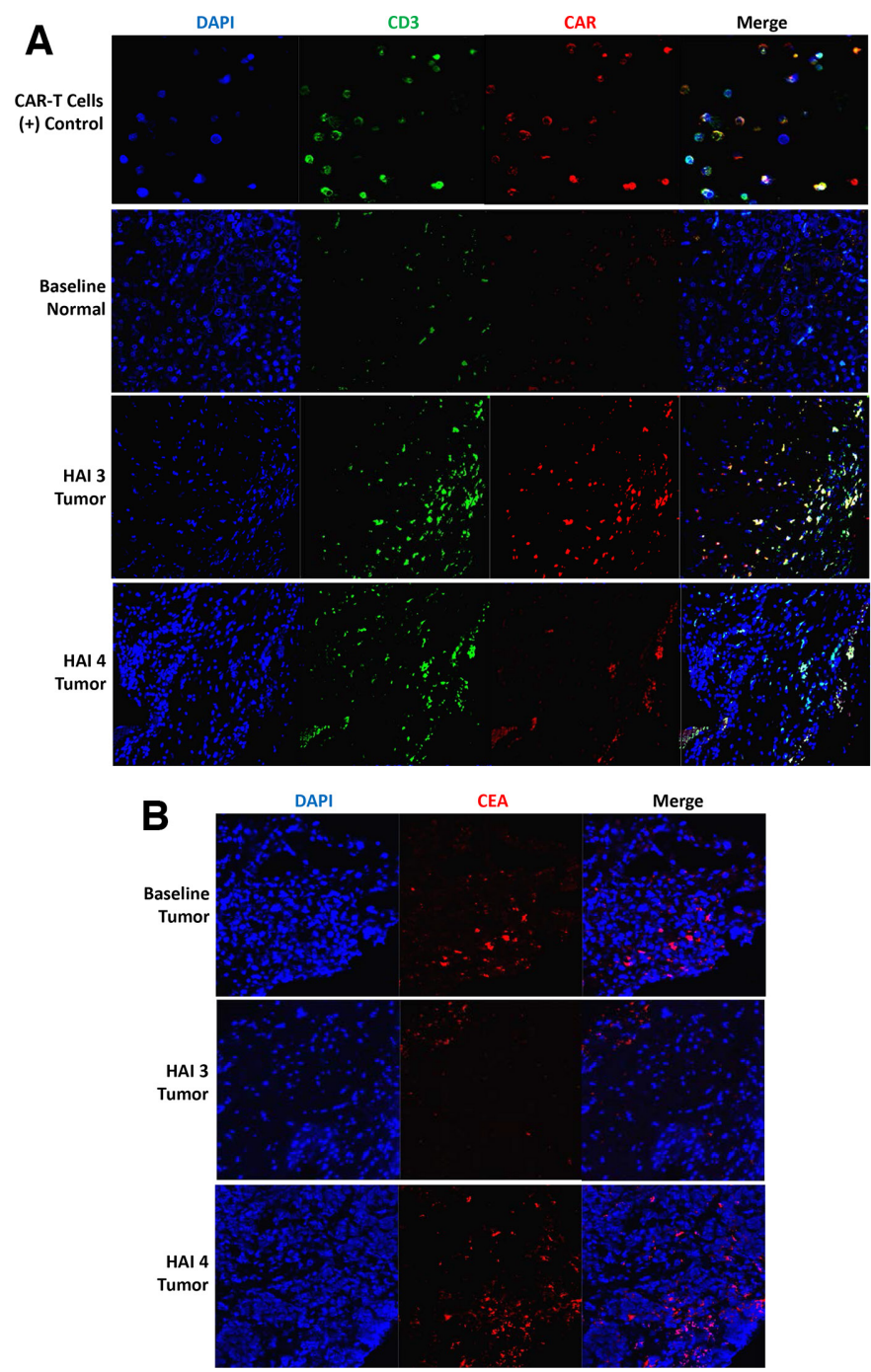
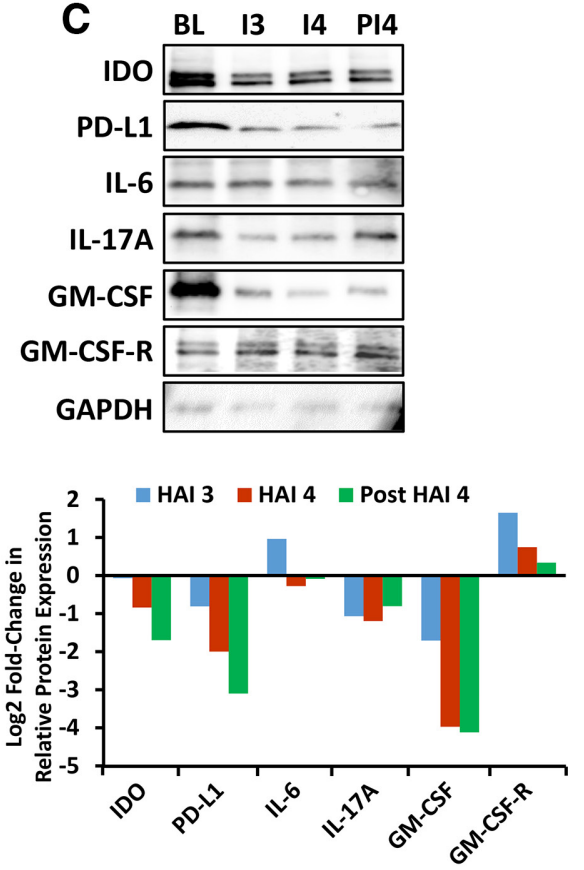

Figure 2 (A) Immunohistochemical analysis of liver tissue for CAR-T detection. Detection of CAR-T in liver tissue by immunofluorescence microscopy showing CAR-T infiltrating tumor post HAI 3 and HAI 4. Liver tissue collected pre and post CAR-T therapy was stained for DAPI, CD3, and CAR. (B) Immunohistochemical analysis of liver tissue for CEA detection. CEA expression in liver tumor tissue detected by immunofluorescence microscopy showing ongoing CEA expression over the course of this study. Liver tumor tissue collected pre and post CAR-T therapy was stained for DAPI and CEA. (C) Change in tumor tissue microenvironment post CAR-T therapy. Lysates of liver tumor tissue collected at baseline (BL), HAl 3 (I3), HAl 4 (I4), and 48 days post HAI 4 (PI4) were analyzed by western blot. Signals were quantified by densitometric analysis and normalized to GAPDH. CAR-T, chimeric antigen receptor T-cells; CEA, carcinoembryonic antigen; HAl, hepatic artery infusion; IL, interleukin, DAPI, 4',6-diamidino-2-phenylindole; IDO, indoleamine 2, 3-dioxygenase; GM-CSF, granulocyte-macrophage colony-stimulating factor; GM-CSF-R, granulocyte-macrophage colony-stimulating factor receptor; GAPDH, glyceraldehyde 3-phosphate dehydrogenase.

pressures, and an immunosuppressive liver milieu represent important obstacles to effective treatment. ${ }^{7}$ In an effort to address these challenges, we have tested high pressure regional infusion approaches whereby CAR-T are infused directly into the liver by HAI using PEDD. Previously we reported that PEDD enhanced CAR-T delivery to LM 5.2-fold when compared with infusion with a standard microcatheter. No grade 4 or 5 AEs related to CAR-T HAIs via PEDD were detected. ${ }^{2}$ This delivery approach is designed to maximize penetration of CAR-T into high pressure solid tumors, while allowing for a more favorable safety profile than is afforded with systemic infusion methods. High-dose regional CAR-T infusions with minimal systemic exposure may lower the risk for cytokine release syndrome and neurologic sequelae. HAI also protects patients from on-target/off-tumor effects, which is particularly important for CAR-T directed against antigens expressed at high levels on normal tissues.

In this subject, HAI of autologous CAR-T targeting CEA mediated a complete metabolic response within the liver which was durable for 13 months. Pathologic analysis of the post-treatment biopsy specimens revealed marked fibrosis in the liver tumor specimen while the integrity of the normal liver tissue was preserved. Western blot analysis of tumor biopsies revealed a shift in the post-treatment tumor microenvironment towards a less 

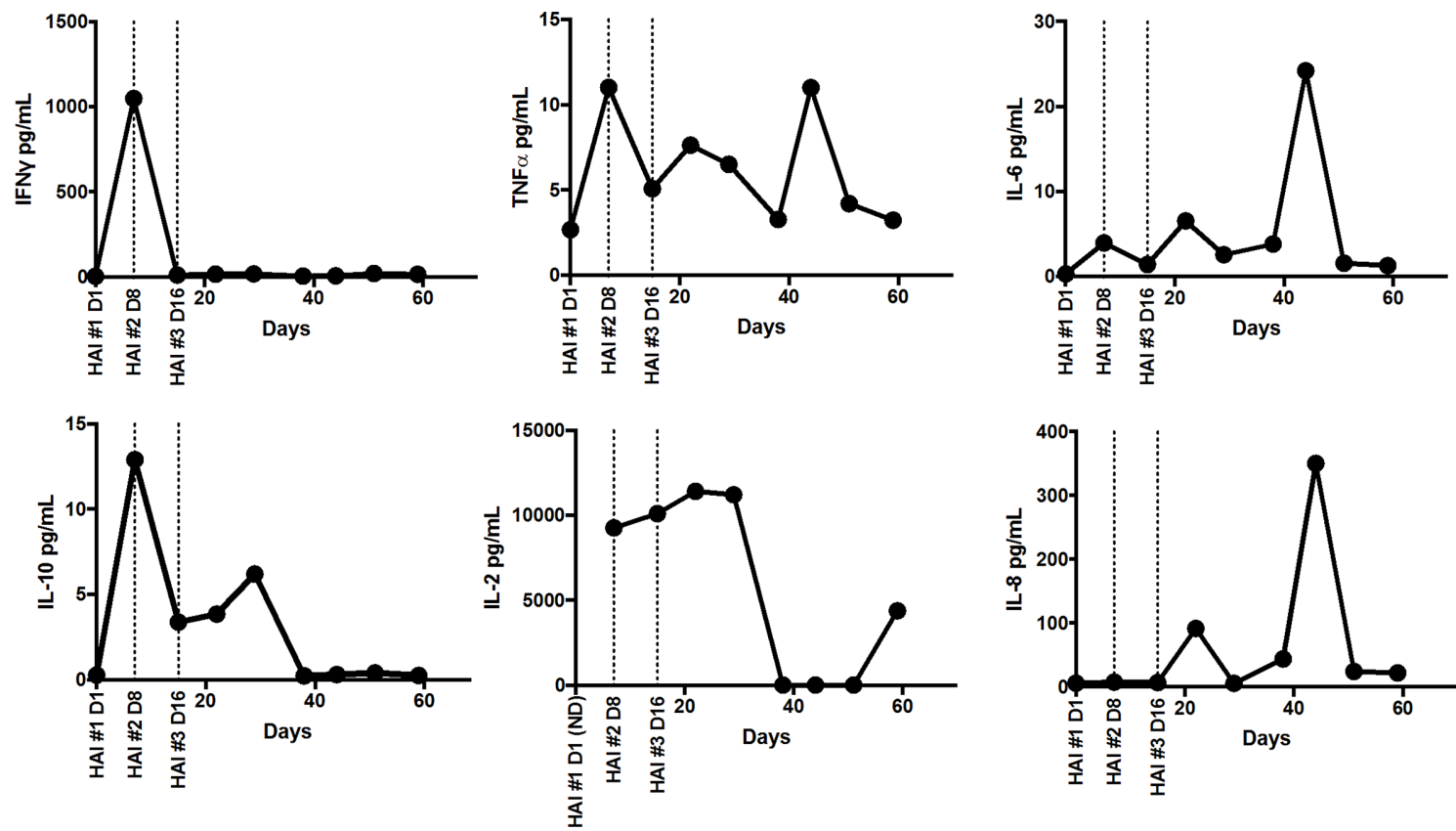

Figure 3 Changes in plasma cytokine levels throughout treatment. Plasma from peripheral blood was collected at baseline and TD 7, 15, 22, 29, 38, 44, 51, and 59 after initiation of treatment. levels of IFNy, TNF $\alpha$, IL-6, IL-10, IL-2, and IL-8 were measured using a multiplex ELISA (human pro-inflammatory V-PLEX, meso scale discovery). Samples were run in duplicate and average cytokine concentrations were extrapolated from an 8-point standard curve. Only samples in the dynamic range of each assay and with CVs below $20 \%$ are shown; IL-2 was below the limit of detection in the baseline samples. IL, interleukin; HAI, hepatic artery infusion; TD, treatment day; IFN, interferon; TNF, tumor necrosis factor; CV, coefficient of variation .

immunosuppressive milieu, characterized by decreased levels of IDO, PD-L1, IL-17A, and GM-CSF. Additionally, a high number of CAR +cells were identified in posttreatment LM tissue. A spike in serum IL-2 was detected following discontinuation of the IL-2 CIVI, suggesting ongoing CAR-T activity, although an isolated increase in serum IL-2 may not be definitive for a sustained antitumor response within the liver. Normalization of tumor markers and their kinetics correlated with the radiographic and pathologic findings observed following treatment. The subject did not respond as well to a subsequent CAR-T HAI when recurrent disease emerged in the liver, possibly due to the absence of systemic IL-2 support and the lower overall CAR-T dose compared with the three CAR-T infusions delivered during the initial treatment. This finding corresponds with data from our previously conducted CAR-T trials, with CEA tumor marker responses occurring in patients who received concurrent IL-2 by CIVI during the CAR-T administration period. ${ }^{14}$ The recurrent tumor maintained CEA expression, ruling out antigen escape.

In this single subject, we demonstrated durable antitumor activity with a low toxicity profile in a patient with stage IV pancreatic cancer resistant to systemic cytotoxic therapy. This patient demonstrated an overall survival time of 23.2 months, compared with the median survival time of 5 months experienced by the majority of stage IV patients. ${ }^{8}$ The findings from this patient, coupled with the data from the previously completed CAR-T HAI trials, ${ }^{14}$ support the excellent safety of this approach with encouraging biologic activity. We envision that HAI of CAR-T may be integrated into the multidisciplinary treatment plan for LM patients, allowing systemic therapies to be administered in sequence when appropriate. This study provides data that regional infusions of anti-CEA CAR-T, in conjunction with CIVI of IL-2, can safely and effectively target CEA-expressing LM and achieve anti-tumor activity in a disease that has proven to be among the most challenging to treat.

\section{Twitter John C Hardaway @jchardaway_md}

Contributors SCK was involved in study conception and design, drafting/revising the manuscript and analysis/interpretation of data. AEM was involved in drafting/ revising/submitting the manuscript and contributed to the figures. PG was involved in performing assays, drafting/submitting the manuscript and contributed to the figures. JCH was involved in drafting the manuscript. EP analyzed radiographic findings and contributed to the figures. JL was involved in performing assays, data acquisition, drafting the manuscript and contributed to the figures. MS analyzed biopsy specimens and contributed to the figures. JES and KRJ were involved in data acquisition, performed cytokine assays and contributed to the figures. RDS and RK were involved in study conception, data analysis and critical review of the manuscript. AS, VA and RPJ were involved in drafting the manuscript.

Funding TriSalus, Sorrento/TNK therapeutics, The State of Colorado Office of Economic Development and Clinigen Group PLC.

Competing interests SCK is an advisor for TriSalus, Nkarta, Takeda, and served as an advisor for TNK Therapeutics during the study. EP serves as a consultant for TriSalus. SCK, JES, KRJ and RDS have received research funding from TriSalus.

\section{Patient consent for publication Not required.}

Ethics approval Human Research Review Committee approval (RW 350-74) and consent was obtained in accordance with local regulations.

Provenance and peer review Not commissioned; externally peer reviewed.

Open access This is an open access article distributed in accordance with the Creative Commons Attribution Non Commercial (CC BY-NC 4.0) license, which permits others to distribute, remix, adapt, build upon this work non-commercially, 
and license their derivative works on different terms, provided the original work is properly cited, appropriate credit is given, any changes made indicated, and the use is non-commercial. See http://creativecommons.org/licenses/by-nc/4.0/.

\section{ORCID iD}

John C Hardaway http://orcid.org/0000-0002-4952-1937

\section{REFERENCES}

1 Katz SC, Burga RA, McCormack E, et al. Phase I hepatic immunotherapy for metastases study of intra-arterial chimeric antigen receptor-modified T-cell therapy for CEA+ liver metastases. Clin Cancer Res 2015;21:3149-59.

2 Katz SC GP, Hardaway JC, Prince E, et al. HITM-SURE: phase Ib CAR-T hepatic artery infusion trial for stage IV adenocarcinoma using Pressure-Enabled drug delivery technology. Washington, D.C: Society of Immunotherapy for Cancer (SITC), 2018.
3 Katz SC, Bamboat ZM, Maker AV, et al. Regulatory T cell infiltration predicts outcome following resection of colorectal cancer liver metastases. Ann Surg Oncol 2013;20:946-55.

4 Katz SC, Hardaway J, Prince E, et al. HITM-SIR: phase Ib trial of intraarterial chimeric antigen receptor T-cell therapy and selective internal radiation therapy for $\mathrm{CEA}^{+}$liver metastases. Cancer Gene Ther 2020;27:341-355.

5 Guha P, Gardell J, Darpolor J, et al. Stat3 inhibition induces Baxdependent apoptosis in liver tumor myeloid-derived suppressor cells. Oncogene 2019;38:533-48.

6 Zarour LR, Anand S, Billingsley KG, et al. Colorectal cancer liver metastasis: evolving paradigms and future directions. Cell Mol Gastroenterol Hepatol 2017;3:163-73.

7 Hardaway JC, Prince E, Arepally A, et al. Regional infusion of chimeric antigen receptor $T$ cells to overcome barriers for solid tumor immunotherapy. J Vasc Interv Radiol 2018;29:1017-21.

8 Ali Al, Oliver AJ, Samiei T, et al. Genetic redirection of T cells for the treatment of pancreatic cancer. Front Oncol 2019;9:56. 